\title{
Respiratory outcomes at five-year follow-up in children with MBL deficiency: a cohort study
}

\author{
Manisha Ramphul ${ }^{1}$, Anna Poghosyan ${ }^{1}$, Javairiya Afzaal ${ }^{1}$, Elizabeth Mcdermott ${ }^{1}$, Lucy \\ Cliffe $^{1}$, and JAYESH MAHENDRA BHATT ${ }^{1}$ \\ ${ }^{1}$ Nottingham University Hospitals NHS Trust
}

May 24, 2021

\begin{abstract}
Introduction Mannose-binding lectin (MBL) serum protein, is an important molecule of the innate immune system that is involved in antimicrobial recognition and clearing responses. There is no conclusive evidence that MBL deficiency is associated with adverse respiratory consequences. Aim We explored whether there is a difference in clinical, radiological and microbiological characteristics in children with MBL deficiency presenting with troublesome respiratory symptoms (frequent, recurrent, persistent or very severe), as compared to those who are MBL-sufficient. Methods We performed a retrospective study looking at MBL measurements in children over a period of 10 years in a large teaching hospital, with a minimum follow-up period of 5 years from the time of the MBL measurement to the year 2019. Results $32 \%$ of children with MBL deficiency and $30 \%$ of those with MBL sufficiency had positive microbiology. $23 \%$ of children with MBL deficiency and $24 \%$ of those with MBL sufficiency had radiological changes on plain radiographs. $28 \%$ of children with MBL deficiency and $33 \%$ of those with MBL sufficiency had suboptimal vaccine responses to primary immunisations. $67 \%$ of the MBL-deficient children had suboptimal vaccine responses to booster immunisations, compared to $40 \%$ of the MBL-sufficient group. Conclusion We conclude that there is no difference at five year follow-up in clinical, radiological and microbiological characteristics between children who are MBL-deficient as compared to those who have sufficient levels. These results add to the existing body of literature that shows no statistically significant association between MBL deficiency and susceptibility to recurrent respiratory tract infection in children.
\end{abstract}

Title page

Title: Respiratory outcomes at five-year follow-up in children with MBL deficiency: a cohort study

Authors

Manisha Ramphul ${ }^{1}$, Anna Poghosyan ${ }^{1}$, Javairiya Afzal ${ }^{1}$, Elizabeth McDermott ${ }^{2}$, Lucy Cliffe ${ }^{1}$, Jayesh M Bhatt $^{1}$

\section{Affiliations}

Department of Paediatric Respiratory Medicine, Nottingham Children's Hospital, Nottingham University Hospitals

Department of Immunology, Nottingham University Hospitals

\section{Correspondence}

Dr Jayesh M Bhatt

Nottingham children's hospital, Queens Medical Centre, Nottingham University Hospitals NHS trust, Derby Rd, Nottingham NG7 2UH

Academic qualifications: MBBS, MD (Paediatrics), DCH (London), FRCPCH 
Email address:jayesh.bhatt@nuh.nhs.uk

Previous presentation of abstract: BTS winter meeting 2021

Keywords : Paediatric, child, pneumonia, respiratory distress, viruses, bacteria, bronchiectasis, immunisation

Word count: 2101 words

Abstract

Introduction

Mannose-binding lectin (MBL) serum protein, is an important molecule of the innate immune system that is involved in antimicrobial recognition and clearing responses. There is no conclusive evidence that MBL deficiency is associated with adverse respiratory consequences.

\section{Aim}

We explored whether there is a difference in clinical, radiological and microbiological characteristics in children with MBL deficiency presenting with troublesome respiratory symptoms (frequent, recurrent, persistent or very severe), as compared to those who are MBL-sufficient.

\section{Methods}

We performed a retrospective study looking at MBL measurements in children over a period of 10 years in a large teaching hospital, with a minimum follow-up period of 5 years from the time of the MBL measurement to the year 2019 .

\section{Results}

$32 \%$ of children with MBL deficiency and $30 \%$ of those with MBL sufficiency had positive microbiology. $23 \%$ of children with MBL deficiency and $24 \%$ of those with MBL sufficiency had radiological changes on plain radiographs. $28 \%$ of children with MBL deficiency and 33\% of those with MBL sufficiency had suboptimal vaccine responses to primary immunisations. $67 \%$ of the MBL-deficient children had suboptimal vaccine responses to booster immunisations, compared to $40 \%$ of the MBL-sufficient group.

\section{Conclusion}

We conclude that there is no difference at five year follow-up in clinical, radiological and microbiological characteristics between children who are MBL-deficient as compared to those who have sufficient levels. These results add to the existing body of literature that shows no statistically significant association between MBL deficiency and susceptibility to recurrent respiratory tract infection in children.

\section{Main body}

Introduction

The innate immune system orchestrates immediate defence against infections. The complement system is a part of the innate immune system and is organised into three distinct enzymatic cascade pathways, namely the classical, alternative and lectin pathways. The lectin pathway is focused on the recognition of repetitive carbohydrate patterns found on the surface of microbial pathogens ${ }^{1}$. The immune response is activated when pathogen associated molecular patterns (PAMPs), displayed on the pathogen surfaces are recognized by cellassociated receptors (pathogen recognising receptors, PRPs) or soluble pathogens recognising molecules (PRMs) such as collectins ${ }^{2}$.

One of the collectins, mannose-binding lectin (MBL), is a liver-produced acute-phase protein and an important first-line defence molecule largely involved in antimicrobial recognition and clearing responses ${ }^{3}$. It activates lectin pathway of complement activation, namely cleaving factor C4 through MBL-associated serine proteases (MASPs) ${ }^{4}$. 
MBL deficiency leads to a primary opsonisation defect; MBL deficiency has hence been associated with severe respiratory infection, due to an impaired host response to infective agents ${ }^{5}$. MBL deficiency predisposes to infection caused by extra-cellular pathogens and activation of autoimmune processes playing crucial role in communicable, inflammatory and autoimmune diseases.

MBL deficiency is common, with more than $10 \%$ of the general population being MBL-deficient ${ }^{6}$. The European Society for Immunodeficiencies guideline ${ }^{1}$ lists MBL deficiency under complement deficiencies and states that data regarding clinical impact of MBL deficiency are contradictory and possible effects include susceptibility to bacterial infections and to autoimmunity. So controversy exists as to when MBL deficiency leads to disease. It has been reported that low MBL levels in children lead to decreased opsonising activity, hence predisposing children to infection ${ }^{7}$.

The levels of MBL vary in the population, with a level of less than $0.6 \mathrm{mg} / \mathrm{L}$ defined as MBL deficiency ${ }^{8}$. At birth, the level is $2 / 3$ of adult level, which increases to the adult level at one month of life, after which the level of MBL within each individual is relatively static and there is a small decrease in later life ${ }^{9}$.

The gene encoding MBL, MBL2, is located on chromosome 10q11.2-q21, consisting of four exons ${ }^{10}$. MBL-2 gene polymorphism is very common; variability is greatly influenced by a type of mutation altering complement activation mechanism involved in the opsonisation process making bacterial clearance less effective ${ }^{11}$.

Investigation of MBL deficiency can be performed by the quantification of serum MBL, or by genotyping for the MBL status ${ }^{6}$. A dilemma with the interpretation of genotyping is that individuals with identical genotypes may differ by 10 -fold in MBL levels ${ }^{10}$.

MBL enhances the respiratory mucosal and bloodstream defenses by binding to respiratory pathogens and promoting $\mathrm{C} 4$ activation. Its deficiency may predispose to respiratory tract infections ${ }^{12}$.

In children with additional risk factors, for example in those with other immuno-deficiencies, the co-existing MBL deficiency increases their propensity to severe infections ${ }^{7}$.

MBL deficiency has also been associated with more severe lung disease in cystic fibrosis $(\mathrm{CF})^{13}$, and also in bronchiectasis not related to $\mathrm{CF}$ in adults ${ }^{14}$; in children with the bronchiectasis not related to $\mathrm{CF}$, there were no significant differences in growth, annual pulmonary exacerbation rates in the last year, pulmonary function tests, radiologic scores, and microbiologic findings between low, intermediate, and high-expressing MBL genotypes ${ }^{15}$.

However in a recent study, MBL levels had no association with infection status at admission, or with progression from systemic inflammatory response syndrome to sepsis or septic shock in children admitted with severe or life-threatening illness ${ }^{16}$. It has also been shown that MBL deficiency is not associated with a susceptibility to influenza-related critical illness in children ${ }^{17}$.

To date, there is no conclusive evidence that MBL deficiency is associated with adverse respiratory consequences at follow-up.

\section{Methods}

We performed a retrospective cohort study looking at the quantification of serum MBL in children over a period of 10 years from 2004 to 2014 at the Nottingham's Children Hospital. This enabled us to define a follow-up period of 5 years or more from the time of the MBL measurement to the year 2019.

Our study was exempt from Institutional Review Board approval, as the MBL serum samples were previously taken for clinical care, and we used the information retrospectively; the subjects cannot readily be identified and we did not need to re-contact and or re-identify the patients.

The inclusion criteria were as follows:

Age less than 18 years

Troublesome respiratory symptoms such as 
frequent or persistent respiratory symptoms such as a chronic wet cough lasting more than 4 weeks recurrent lower respiratory tract infections ([?]4 infections in a year)

severe respiratory tract infections requiring admission to intensive care or to the high dependency unit.

The MBL level was checked as part of baseline immunology testing to exclude a primary immune deficiency.

MBL deficiency was defined as a serum level $<0.6 \mathrm{mg} / \mathrm{L}$. The difference in clinical, radiological and microbiological characteristics in patients with MBL deficiency in children presenting with troublesome respiratory symptoms (frequent, recurrent, persistent or very severe) was compared to those with MBL-sufficient levels.

We defined a suboptimal response to primary vaccinations based on antibody titres, which were below the laboratory quoted ranges for Haemophilus influenzae $B$ and tetanus. For Prevenar, the response was deemed as suboptimal response when fewer than 8 serotypes were at the desired level.

\section{Statistical analysis}

The data was analysed on GraphPad Prism 9. We tested for normality, and non-normally distributed data were expressed as median and interquartile ranges. The Chi-squared test was used to compare the followup parameters of MBL-deficient and MBL-sufficient children. The Odds Ratio (OR), the $95 \%$ confidence intervals (CI) and p-values were calculated. A p-value $<0.05$ was considered as statistically significant.

\section{Results}

206 patients undergoing MBL testing were included in the study, out of whom 43 (21\%) were MBL-deficient.

The baseline characteristics of the study population are shown below in Table 1 .

\section{Table 1: Baseline characteristics of study population}

The clinical, radiological and microbiological characteristics of the MBL-deficient and MBL-sufficient children are summarised in the Table 2. There were no clinical or statistical differences between the two groups.

Table 2: Clinical, radiological and microbiological parameters over follow-up period

The various follow-up parameters are depicted in Figure 1.

Figure 1: Bar chart showing follow-up features of $M B L$-deficient and MBL-sufficient children

The percentage of children requiring admission to PICU was small and similar in two groups. $28 \%$ of children with MBL deficiency and 33\% of those with MBL sufficiency had suboptimal vaccine responses to primary immunisations.

$32 \%$ of children with MBL deficiency and $30 \%$ of those with MBL sufficiency had positive microbiology (respiratory viruses, bacteria or both).

$23 \%$ of children with MBL deficiency and $24 \%$ of those with MBL sufficiency had radiological changes on plain radiographs. We performed a subgroup analysis to look at presence of bronchiectasis in children undergoing a CT chest. Five MBL-deficient children and 10 MBL-sufficient children underwent a CT scan and the proportion of bronchiectasis was similar in both groups, as shown in Figure 2.

Figure 2: Chart showing risk of bronchiectasis in the two groups

Subgroup analysis- booster immunisations

Our data did not show in a difference in adequacy of response to primary immunisations in the MBL-deficient cohort, compared to the MBL-sufficient cohort. Further subgroup analysis noted that children with MBL deficiency were clinically less likely to respond well to booster immunisations (67\% of the MBL-deficient children compared to $40 \%$ of MBL-sufficient children), as shown in Figure 3. However, the difference was not statistically significant (p-value 0.08) due to small numbers. 
Figure 3: Adequacy of response to booster immunisations in MBL-deficient and MBL-sufficient children

Discussion

Different studies have explored the relationship between MBL levels and increased susceptibility to respiratory infections both in children and adults, with different conclusions ${ }^{18,19}$. Even if children do have an increased susceptibility to respiratory infections, our study found no adverse clinical, radiological or microbiological consequences in these children at 5 years follow up.

On one hand, the literature suggests that children with another immunodeficiency and MBL deficiency may be more prone to infections ${ }^{5}$. It has been suggested that MBL deficiency plays an important role in host defence, especially during the vulnerable period of infancy between 6 and 17 months of age, when maternal antibodies wane and the adaptive immune system is still developing ${ }^{18}$. These factors make children susceptible to infection by specific pathogens such as $S$. pneumoniae - one of the most important pathogens causing respiratory tract infections ${ }^{20}$.

The association between MBL gene polymorphism and respiratory infections was confirmed in children admitted to a hospital with either Upper Respiratory Tract infections (URTIs) or alternative diagnoses. More severe infections including recurrent URTI were noted in children with homozygous mutation of MBL2 gene, and the same trend was observed at 2 year follow-up ${ }^{21}$.

A recent meta-analysis shows that MBL deficiency is associated with death in patients with pneumococcal infection after adjusting other confounders ${ }^{22}$. Neonates with low MBL levels have been deemed to be at higher risk of early-onset sepsis and pneumonia ${ }^{23}$, and children with low MBL levels have often been reported to be at higher risk of severe infections ${ }^{24}$.

On the other hand, we observed no association between MBL deficiency and susceptibility to pneumococcal community-acquired pneumonia and invasive pneumococcal disease ${ }^{19}$. One of the recent studies has shown no relation between MBL deficiency and severe lower respiratory tract infection caused by respiratory syncytial virus $^{25}$. These findings support the findings of our study.

It is worth mentioning that most of MBL-disease association studies to date have assessed MBL2 genotypes with or without MBL levels, with MBL insufficient genotypes being $\mathrm{XA} / \mathrm{O}$ or $\mathrm{O} / \mathrm{O}^{25}$.

However, changes in MBL gene expression associated with low MBL protein levels have been found in healthy individuals commonly and may vary when measured in disease as an acute-phase protein ${ }^{26}$. Emerging studies identified no existing association between MBL deficiency and recurrent URTI such as otitis media in children. These data suggest that low MBL concentration does not necessarily indicate a propensity to disease ${ }^{27}$ and low levels have to be interpreted alongside clinical features and existing symptoms.

Thus, the association between MBL protein levels and recurrent respiratory infections in children remains debatable. The link between MBL polymorphisms and respiratory tract infections in children remains controversial $^{21}$. Confounders such as atopy, parental smoking and environmental factors, as well as small sample sizes of the studies, make it difficult to attribute the cause solely to MBL deficiency.

Hence MBL testing performed as part of baseline immunology testing in this group of children may be unnecessary, as the results seem to neither guide the management, nor the prognosis. This recommendation would be in line with the recommendations of the recent evidence-based ERS guidelines ${ }^{28}$, and result in more cost-effective practice, given that each MBL level assay costs $£ 24(\$ 34)^{29}$.

Whilst our study showed that children with MBL deficiency were more likely to respond inadequately to appropriate booster immunisations, a previous study did not find an association between MBL deficiency and response to pneumococcal vaccination ${ }^{30}$. Equally, MBL polymorphisms have been shown not to affect production and persistence of antibodies for acellular pertussis ${ }^{31}$. Longer term prospective studies may shed more light on this area.

Strengths and limitations 
The most important strength of the study is the size of the cohort, with the inclusion of a large number of children, and a substantial period of follow up of five years or longer. Our study is hence a robust addition to the existing evidence regarding the specifics of respiratory infections in children with MBL deficiency.

Our study had a number of limitations. It was a retrospective study, which may introduce selection and recall bias. Whilst undertaking the subgroup analyses, our subgroup numbers were relatively low. Also, we did not include a symptom score and hence could not quantify the degree of symptoms that children with MBL deficiency and sufficiency subjectively experienced. Finally we defined MBL deficiency on biochemical values, rather than by genetic polymorphisms, and ideally both measures would have been included.

\section{Conclusion}

To conclude, we showed no difference at five year follow-up in clinical, radiological and microbiological characteristics between children who are MBL-deficient, compared to those who have sufficient levels. The rates of admission to PICU were similar in the two groups. About a quarter of children we followed up had long term pulmonary changes on the CXR and the prevalence of bronchiectasis was similar in both groups. Children with MBL deficiency in our cohort are more likely to respond poorly to appropriate booster immunisation, but the significance of this is unclear.

These results add to the existing body of literature that shows no statistically significant association between MBL deficiency and susceptibility to recurrent respiratory tract infection in children. Additionally the results show no adverse respiratory consequences at 5 year follow up in those with MBL deficiency as compared to those children who are MBL-sufficient.

\section{References}

1. Brodszki N, Frazer-Abel A, Grumach AS, et al. European Society for Immunodeficiencies (ESID) and European Reference Network on Rare Primary Immunodeficiency, Autoinflammatory and Autoimmune Diseases (ERN RITA) Complement Guideline: Deficiencies, Diagnosis, and Management. J Clin Immunol . 2020;40(4):576-591. doi:10.1007/s10875-020-00754-1

2. Jack DL, Turner MW. Anti-microbial activities of mannose-binding lectin. Biochem Soc Trans . 2003;31(4):753-757. doi:10.1042/bst0310753

3. Takahashi K, Ezekowitz RAB. The Role of the Mannose-Binding Lectin in Innate Immunity. Clin Infect Dis . 2005;41(Supplement_7):S440-S444. doi:10.1086/431987

4. Thiel S, Møller-Kristensen M, Jensen L, Jensenius JC. Assays for the Functional Activity of the MannanBinding Lectin Pathway of Complement Activation. Immunobiology . 2002;205(4):446-454. doi:10.1078/0171$2985-00145$

5. Cedzynski M, Szemraj J, Swierzko AS, et al. Mannan-binding lectin insufficiency in children with recurrent infections of the respiratory system. Clin Exp Immunol . 2004;136(2):304-311. doi:10.1111/j.13652249.2004.02453.x

6. Thiel S, Frederiksen PD, Jensenius JC. Clinical manifestations of mannan-binding lectin deficiency. Mol Immunol . 2006;43(1-2):86-96. doi:10.1016/j.molimm.2005.06.018

7. Super M, Lu J, Thiel S, Levinsky RT, Turner MW. ASSOCIATION OF LOW LEVELS OF MANNAN-BINDING PROTEIN WITH A COMMON DEFECT OF OPSONISATION.The Lancet . 1989;334(8674):1236-1239. doi:10.1016/S0140-6736(89)91849-7

8. Mannose binding lectin. Available from https://www.ouh.nhs.uk/immunology/diagnostic-tests/testscatalogue/mbl.aspx. Last accessed on 22 November 2020.

9. Ip WK, To YF, Cheng SK, Lau YL. Serum Mannose-Binding Lectin Levels and mbl2 Gene Polymorphisms in Different Age and Gender Groups of Southern Chinese Adults. Scand J Immunol . 2004;59(3):310-314. doi:10.1111/j.0300-9475.2004.01392.x 
10. Steffensen R, Thiel S, Varming K, Jersild C, Jensenius JC. Detection of structural gene mutations and promoter polymorphisms in the mannan-binding lectin (MBL) gene by polymerase chain reaction with sequence-specific primers. J Immunol Methods . 2000;241(1):33-42. doi:10.1016/S0022-1759(00)00198-8

11. Summerfield JA, Sumiya M, Levin M, Turner MW. Association of mutations in mannose binding protein gene with childhood infection in consecutive hospital series. BMJ . 1997;314(7089):1229. doi:10.1136/bmj.314.7089.1229

12. Eisen DP. Mannose-binding lectin deficiency and respiratory tract infection. J Innate Immun . 2010;2(2):114-122. doi:10.1159/000228159

13. Chalmers JD, Fleming GB, Hill AT, Kilpatrick DC. Impact of mannose-binding lectin insufficiency on the course of cystic fibrosis: A review and meta-analysis. Glycobiology . 2011;21(3):271-282. doi:10.1093/glycob/cwq161

14. Chalmers JD, McHugh BJ, Doherty C, et al. Mannose-binding lectin deficiency and disease severity in non-cystic fibrosis bronchiectasis: a prospective study. Lancet Respir Med . 2013;1(3):224-232. doi:10.1016/S2213-2600(13)70001-8

15. Dogru D, Polat SE, Tan Ç, et al. Impact of mannose-binding lectin 2 gene polymorphisms on disease severity in noncystic fibrosis bronchiectasis in children. Pediatr Pulmonol . 2020;55(5):1190-1198. doi:10.1002/ppul.24711

16. Madsen EC, Levy ER, Madden K, et al. Mannose-Binding Lectin Levels in Critically Ill Children With Severe Infections. Pediatr Crit Care Med J Soc Crit Care Med World Fed Pediatr Intensive Crit Care Soc . 2017;18(2):103-111. doi:10.1097/PCC.0000000000001000

17. Levy ER, Yip W-K, Super M, et al. Evaluation of Mannose Binding Lectin Gene Variants in Pediatric Influenza Virus-Related Critical Illness. Front Immunol . 2019;10:1005. doi:10.3389/fimmu.2019.01005

18. Koch A, Melbye M, Sørensen P, et al. Acute Respiratory Tract Infections and Mannose-Binding Lectin Insufficiency During Early Childhood. JAMA . 2001;285(10):1316-1321. doi:10.1001/jama.285.10.1316

19. García-Laorden MI, Rodríguez de Castro F, Solé-Violán J, et al. The role of mannose-binding lectin in pneumococcal infection. Eur Respir J . 2013;41(1):131. doi:10.1183/09031936.00174111

20. Darmawan AB, Soesatyo MHNE, Restuti RD, Surono A. The Role of Mannose-Binding Lectin Serum Level in Tubotympanic Chronic Suppurative Otitis Media. Int J Otolaryngol . 2018;2018:6178159-6178159. doi:10.1155/2018/6178159

21. Ruskamp JM, Hoekstra MO, Rovers MM, Schilder AGM, Sanders EAM. Mannose-Binding Lectin and Upper Respiratory Tract Infections in Children and Adolescents: A Review. Arch Otolaryngol Neck Surg . 2006;132(5):482-486. doi:10.1001/archotol.132.5.482

22. Eisen DP, Dean MM, Boermeester MA, et al. Low Serum Mannose-Binding Lectin Level Increases the Risk of Death due to Pneumococcal Infection. Clin Infect Dis . 2008;47(4):510-516. doi:10.1086/590006

23. Frakking FNJ, Brouwer N, van Eijkelenburg NKA, et al. Low mannose-binding lectin (MBL) levels in neonates with pneumonia and sepsis. Clin Exp Immunol . 2007;150(2):255-262. doi:10.1111/j.13652249.2007.03479.x

24. Taras R, Stefan M, Derewicz D, Ionescu M, Cinteza E, Balgradean M. Characteristics of MannoseBinding Lectin Deficiency in Pediatric Septic Patients - Case Presentation. Maedica . 2019;14(1):49-52. doi:10.26574/maedica.2019.14.1.49

25. Kristensen IA, Thiel S, Steffensen R, Madhi S, Sorour G, Olsen J. Mannan-Binding Lectin and RSV Lower Respiratory Tract Infection Leading to Hospitalization in Children: A Case-Control Study from Soweto, South Africa. Scand J Immunol . 2004;60(1-2):184-188. doi:10.1111/j.0300-9475.2004.01466.x 
26. Dean MM, Minchinton RM, Heatley S, Eisen DP. Mannose Binding Lectin Acute Phase Activity in Patients with Severe Infection. J Clin Immunol . 2005;25(4):346-352. doi:10.1007/s10875-005-4702-1

27. Homoe P, Madsen H, Sandvej K, Koch A, Garred P. Lack of association between mannose-binding lectin, acute otitis media and early Epstein-Barr virus infection among children in Greenland. Scand J Infect Dis . 1999;31(4):363-366. doi:10.1080/00365549950163798

28. Chang AB, Fortescue R, Grimwood K, et al. Task Force report: European Respiratory Society guidelines for the management of children and adolescents with bronchiectasis. Eur Respir J . Published online January 1, 2021:2002990. doi:10.1183/13993003.02990-2020

29. Sheffield Teaching Hospitals NHS Trust, Directorate of Laboratory Medicine; 2019. Mannose Binding Lectin

30. van Kessel DA, Hoffman TW, van Velzen-Blad H, Zanen P, Rijkers GT, Grutters JC. Response to pneumococcal vaccination in mannose-binding lectin-deficient adults with recurrent respiratory tract infections. Clin Exp Immunol . 2014;177(1):272-279. doi:10.1111/cei.12299

31. Grondahl-Yli-Hannuksela K, Vuononvirta J, Peltola V, Mertsola J, He Q. Lack of association between mannose binding lectin and antibody responses after acellular pertussis vaccinations. PloS One . 2014;9(2):e88919-e88919. doi:10.1371/journal.pone.0088919

\section{Hosted file}

Table 1.docx available at https://authorea.com/users/415440/articles/523326-respiratoryoutcomes-at-five-year-follow-up-in-children-with-mbl-deficiency-a-cohort-study

\section{Hosted file}

Table 2.docx available at https://authorea.com/users/415440/articles/523326-respiratoryoutcomes-at-five-year-follow-up-in-children-with-mbl-deficiency-a-cohort-study

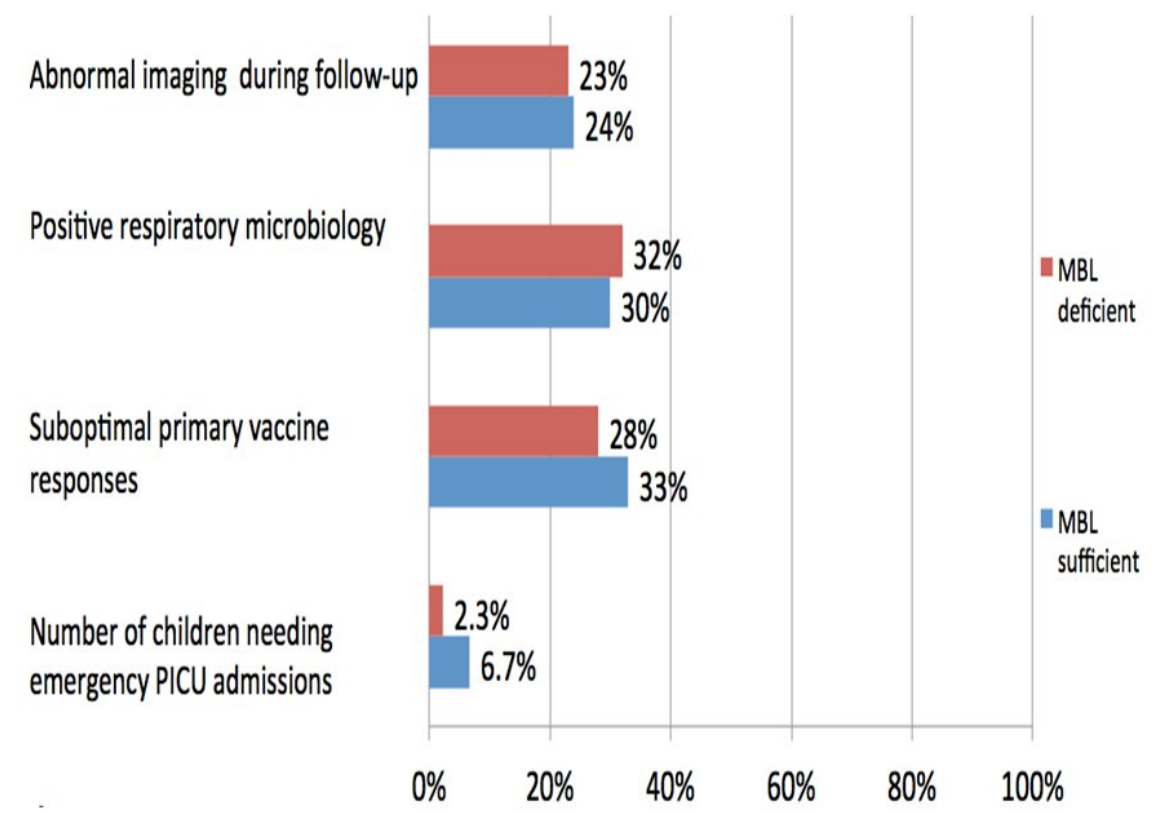




\section{Risk of bronchiectasis}

$5 \mathrm{MBL}$ deficient children underwent a CT chest

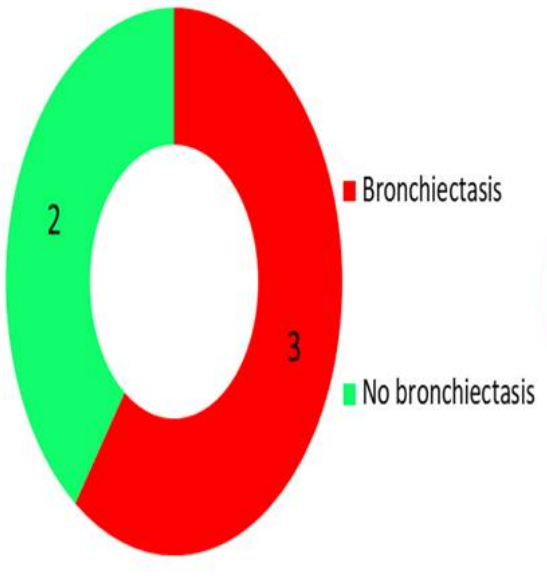

$10 \mathrm{MBL}$ sufficient children underwent a $\mathrm{CT}$ chest

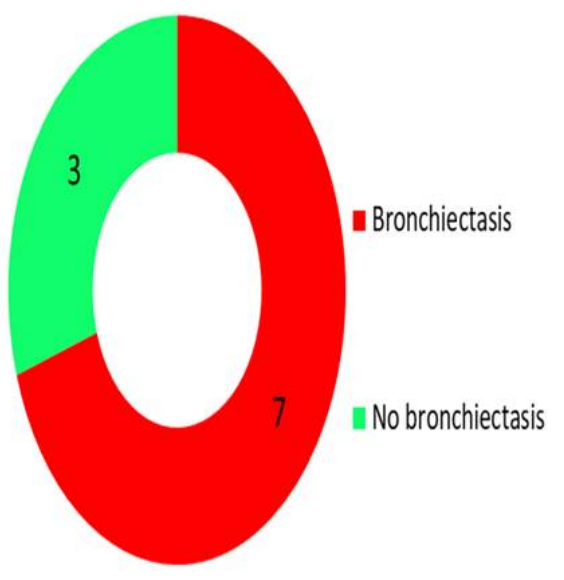

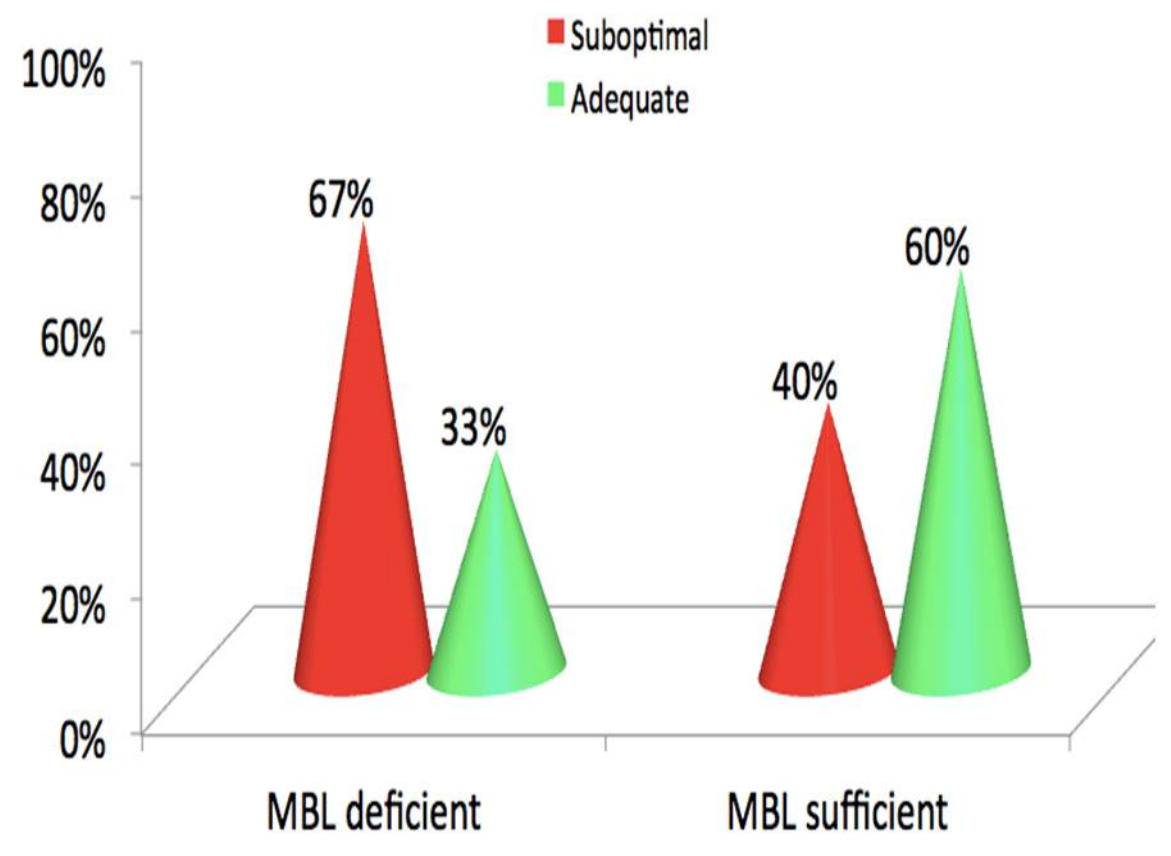

